\title{
EFFECT OF WEAR ON THE MAGNETIC PROPERTIES AND TENSILE STRENGTH OF STEEL WIRE
}

\author{
By Raymond L. Sanford, Walter L. Cheney, and James M. Barry
}

ABSTRACT

The work here described is part of a more extended investigation on nondestructive methods of testing wire rope. In order to design intelligently apparatus for the nondestructive testing of wire rope it is necessary to know the nature and magnitude of the effects involved. One of the causes of deterioration in wire rope is wear, and this paper is a description of the work done to determine the effect of wear on the magnetic properties and tensile strength of steel wire of the type used in the manufacture of wire rope.

It was found that wear increases the magnetic permeability for low values of magnetizing force and decreases it for higher forces. This effect is similar to the effect of stress, though much less in magnitude, and is attributed to a redistribution of internal stress. This change in magnetic properties is accompanied by an increase in tensile strength.

\section{CONTENTS.}

I. Statement of the problem

339

II. Material and apparatus_......... 340

III. Experimental results _._.

IV. Summary and conclusions_._. 344

\section{STATEMENT OF THE PROBLEM}

The Bureau of Standards has been engaged for some time upon an investigation of the possibility of applying the methods of magnetic analysis to the nondestructive testing of wire hoisting rope. The primary object of this investigation is to determine whether or not a practical device can be developed for testing hoisting ropes in place and under service conditions. In previous investigations on the detection of flaws and internal defects, it has been found that, while such flaws and defects are always indicated by a change in magnetic permeability, similar magnetic effects are often observed which can not be correlated with flaws or defects within the material. These disturbing effects may be greater in magnitude than those caused by the flaws it is wished to locate, and the resulting unreliability of the results places a serious limitation upon the practical applicability of the magnetic method for the detection of flaws. With this fact in mind, the present investigation was undertaken with a view to the discovery of the causes of uncertainty in the results and the establish- 
ment of the fundamental principles underlying the observed phenomena. The effects of the main causes of deterioration in hoisting rope on the magnetic properties are being studied separately. These factors include wear, fatigue, overstrain, and corrosion. The effect of mechanical stress on magnetic properties has also been studied, because stress, both internal and external, is always present in wire rope under service conditions. It has been found ${ }^{1}$ that this factor is responsible to a large degree for the uncertainty in the interpretation of results and must always be taken into consideration. It is the object of this paper to describe the experiments which have been made to determine the effects of wear on the magnetic properties and mechanical strength of hard-drawn steel wire.

\section{MATERIAL AND APPARATUS}

The material tested was crucible steel wire ${ }^{2} 0.1$ inch in diameter of the following composition:

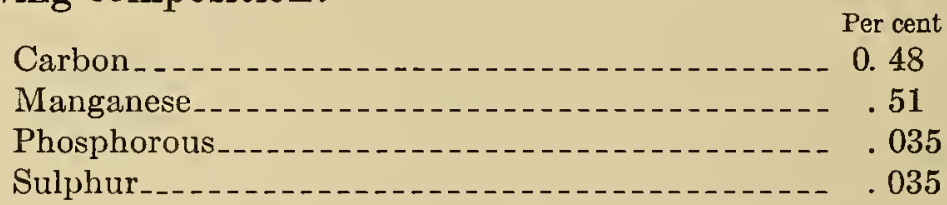

This was patented wire, of the type ordinarily used in the construction of hoisting rope, having an ultimate strength of approximately 195,000 lbs./in. ${ }^{2}$ Wire was used, instead of the finished rope, because it is simpler in form, and the results are easier of interpretation.

Figure 1 shows the apparatus used to wear down the wire. This machine consists of a main frame or track, with clamps at each end for holding the wire under test, and upon which runs a carriage. Mounted on this carriage is an electric motor which serves the double purpose of operating the wearing wheel and of carrying it back and forth along the length of the wire. The wearing wheel, of cold-rolled steel, ${ }^{3}$ is mounted directly on the armature shaft of the motor which runs at a speed of about 2,400 revolutions per minute. The plane of the wheel is parallel to the length of the wire, which is held against it by a hardened steel shoe, the pressure being regulated by an adjustable spring. The carriage is driven by a rack and pinion through a reduction gear, which is integral with the motor. A reversing switch, operated by stops at either end of the main frame, reverses the direction of travel. At one end of the apparatus is a ratchet by means of which the wire is rotated about its own axis $1 / 40$ of a revolution for each round trip of the carriage. Undue heating of the wire is avoided by using a light pressure and by a current of air from an

1 R. L. Sanford, B. S. Sci. Paper, No. 495; 1921

2 Furnished for this investigation by the American Steel \& Wire Co.

${ }^{3}$ Cast-iron wheels and alundum grinding wheels were tried, but it was found that the steel wheel gave the most unitorm and satisfactory results. 


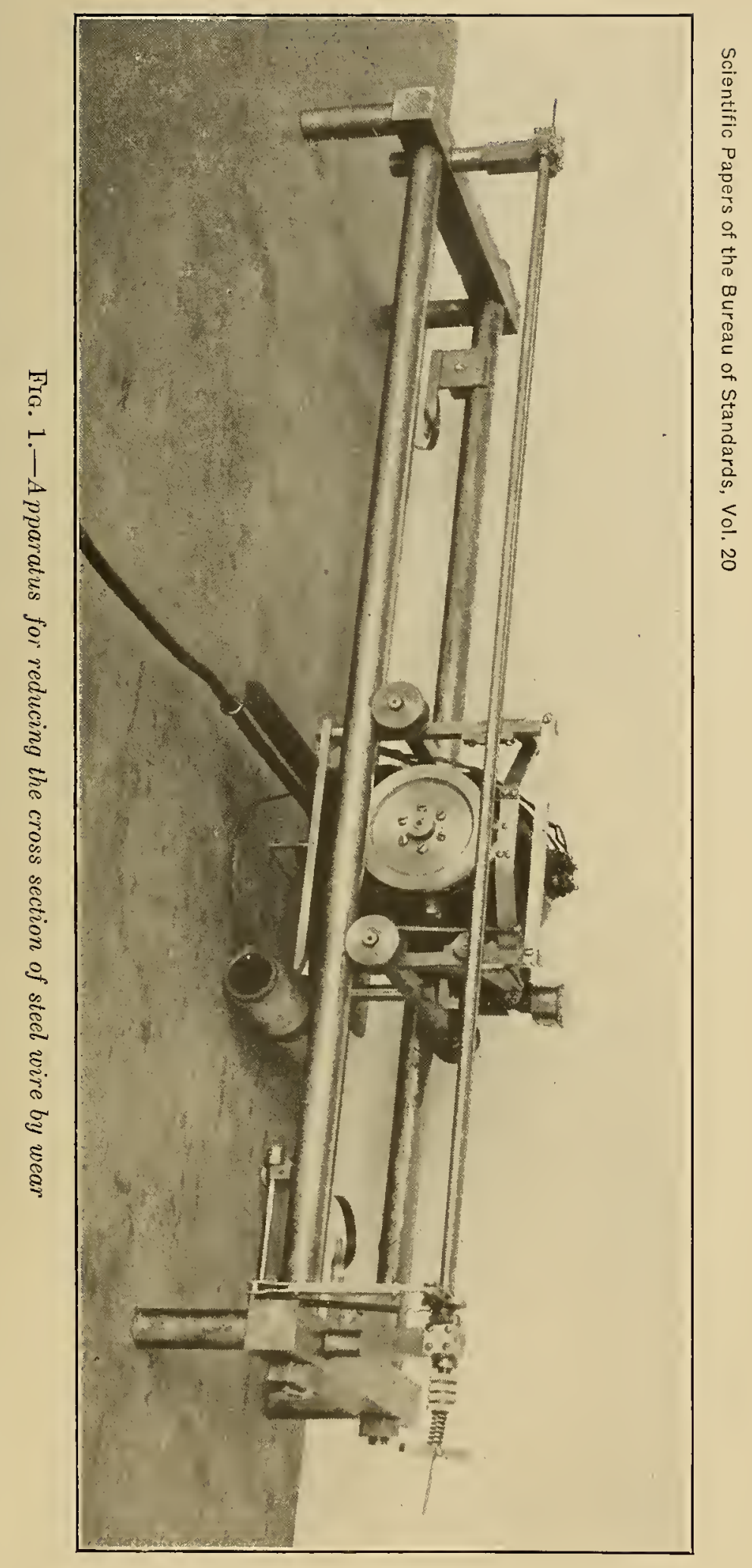


electric fan. During the operation of the machine it is possible to rest the hand upon the wire without discomfort.

With this machine, the diameter of a wire could be reduced with satisfactory uniformity over a length of about $60 \mathrm{~cm}$. The machine proved to be sufficiently reliable in operation to permit of its running, unattended, during the night, and a simple automatic stop was devised which operated after a predetermined number of complete revolutions of the wire about its axis. This resulted in a considerable saving of time. ${ }^{4}$

The magnetic measurements were made by the ordinary ballistic method. The samples were magnetized in a straight solenoid $50 \mathrm{~cm}$ long capable of producing magnetizing forces up to 1,000 gilberts per $\mathrm{cm}$. The winding of this coil is such that the magnetizing force is approximately 100 times the current in amperes. As the ratio of length to diameter of the samples was over 200, the demagnetizing factor could be neglected. The test coils, of 100 turns each, were wound directly on the specimens so as to avoid a large correction for air flux. The ballistic galvanometer was calibrated by means of a standard mutual inductance whose secondary was kept always in the galvanometer circuit. The galvanometer was heavily overdamped and gave practically fluxmeter performance.

For the mechanical tests, a Scott horizontal testing machine of 2,000 pounds capacity was used.

\section{EXPERIMENTAL RESULTS}

The experimental procedure was to determine normal induction data for an unworn sample of wire and then to repeat the magnetic test after successive reductions in diameter in the wear machine. It was found that the effect of wear, though much smaller in magnitude, was similar in nature to that of an externally applied tensile stress. For the lower values of magnetizing force, the magnetic permeability is increased. The curves cross, however, and there is a decrease in permeability for the higher values of magnetizing force. This effect is illustrated in the curves of Figure 2. The results shown in the figure ${ }^{5}$ were obtained for a reduction in area of 16.5 per cent. Results of a similar nature, though varying in magnitude, were obtained for other amounts of reduction in area.

There is some uncertainty in the results, due to the strain effects resulting from inserting a sample of wire, originally curved, in the magnetizing solenoid. It was found, however, that by putting the samples in a fiber tube one-fourth inch in diameter before placing them in the solenoid, fairly consistent results could be obtained. No further attempt to eliminate this variation was made because it

\footnotetext{
${ }^{4}$ Credit is due to H. J. Hoyert for many details in the design and operation of this machine.

${ }^{5}$ The curve $\triangle B$ showing change in induction being given because this change, though real, is so small that it can not be shown satisfactorily on the same scale as that used for the $B-H$ curve.
} 
was considered sufficient for the purpose to know the general nature and approximate magnitude of the effect.

It was found that in all cases the maximum change in induction, due to wear, occurred at a magnetizing force of 20 gilberts per $\mathrm{cm}$. The relation between this maximum change and the per cent reduction in area is shown in Figure 3.

The curves of Figures 2 and 3 indicate that the effect of wear on the magnetic properties of the material under consideration differs

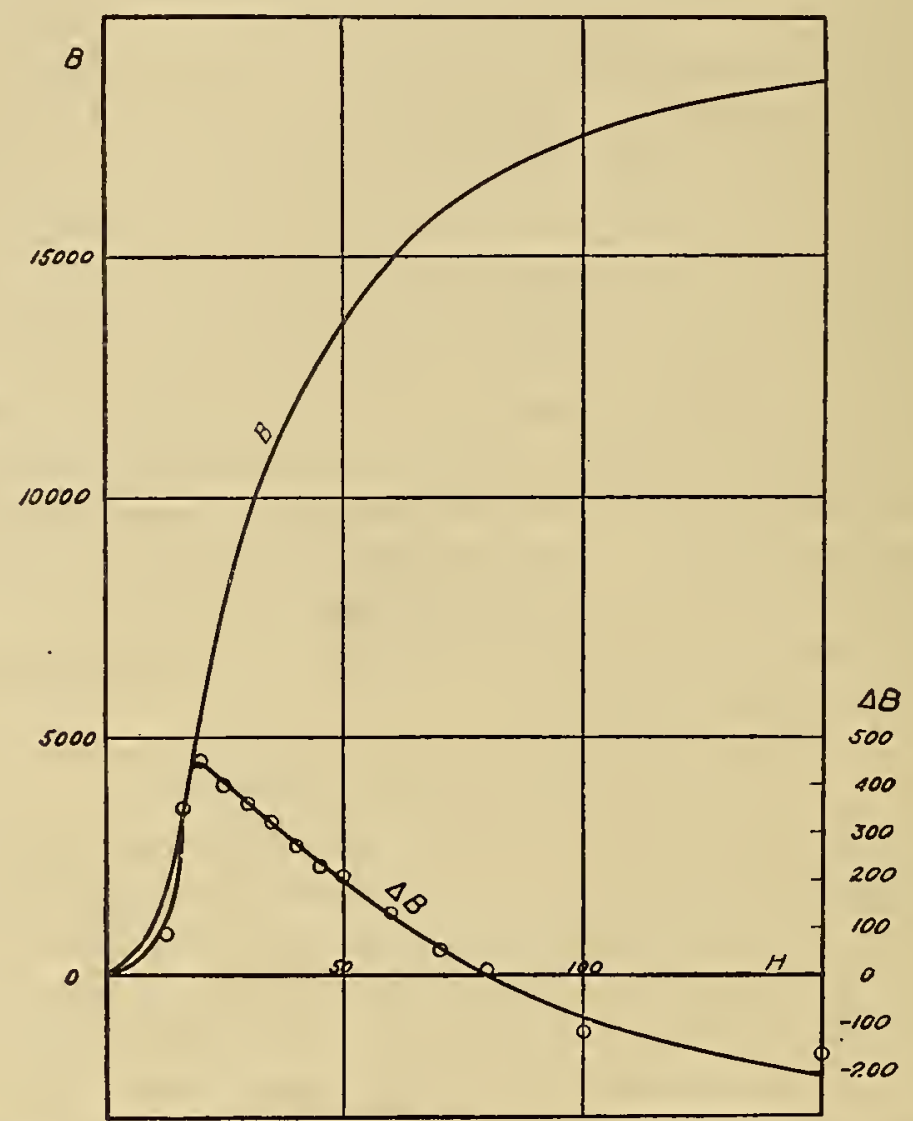

FIG. 2.-Effect of reduction in area by wear on the magnetic properties of steel wire only in magnitude from the effect of a tensile stress. Indeed, it seems reasonable to conclude that they arise from the same source, namely, a change in stress distribution within the material. If we consider that the outer part of the wire is in a state of tension and that this is a very small fraction of the whole, then the bulk of the material must be under compression and the magnetic properties will correspond practically to those of the compression material. The removal of some of the tension material results in an elongation with a corresponding decrease in the degree of compression in the bulk of the material, thus producing a magnetic effect somewhat similar to that of an elongation produced by an external load. There is this important difference, however. In the case of an external force the total stress in the material is increased, whereas the total 
stress is decreased by wear. It would naturally be expected, therefore, that the effect of wear would be smaller than that of tensile stress.

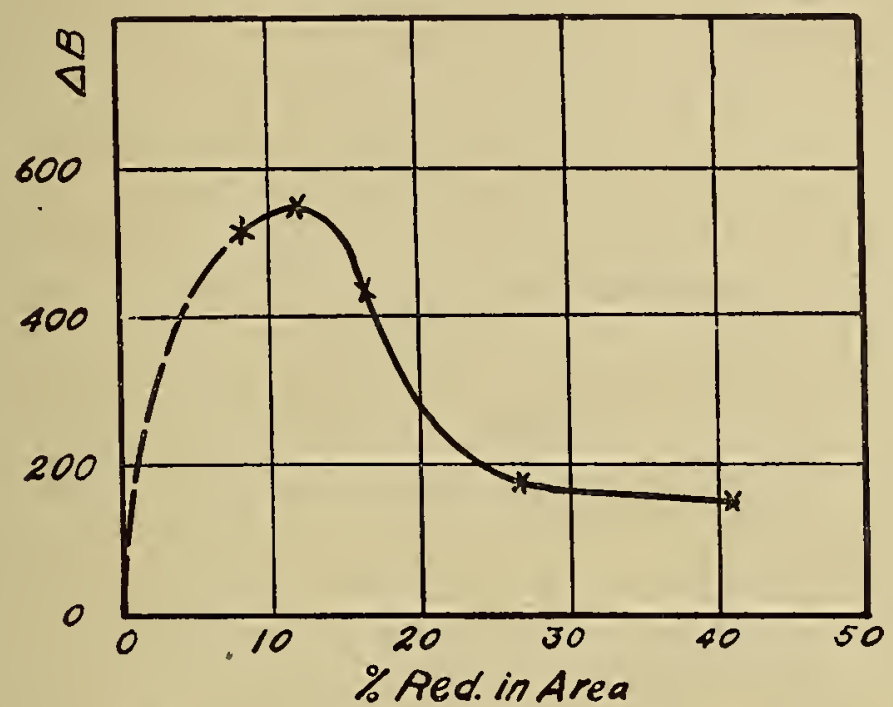

Frg. 3.-Relation between per cent reduction in area by wear and change in mag-
netic induction for $H=20$

If the foregoing explanation is valid, we should find that the mechanical effect of wear on this material would be an increasein strength per unit area of cross section. In order to test this, a number of wires were worn down to different diameters in the wear machine and tested for ultimate strength. The results are indicated in Figure 4. The exact form of the curve is not known, as the points represent

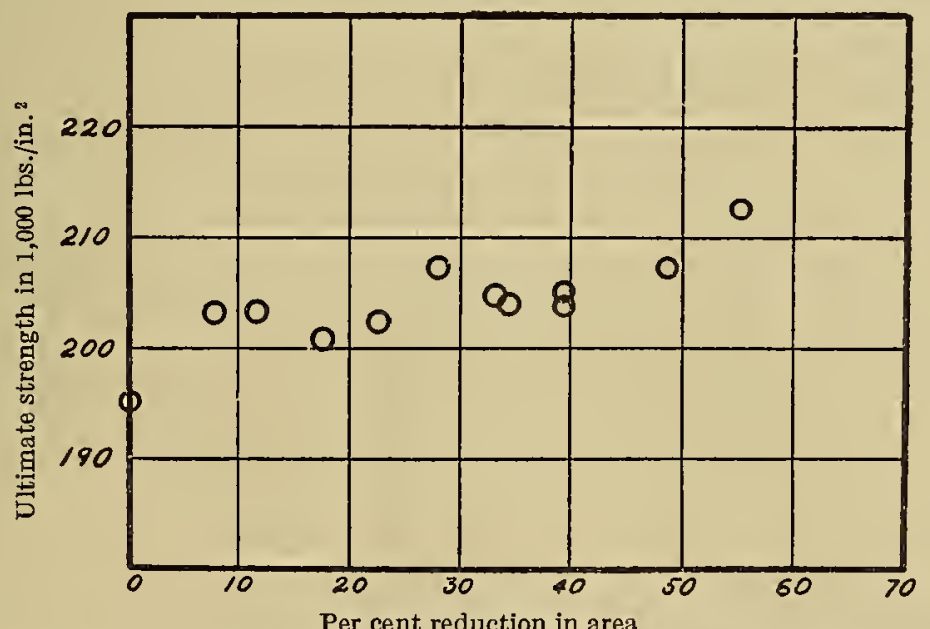

FIG. 4.-Increase in tensile strength of steel wire resulting from reduction in area by wear

single determinations. However, a distinct and progressive increase in ultimate strength per unit area with reduction in area is noted. This increase in strength probably results from a redistribution of initial internal stress which renders a greater proportion of the material effective. 


\section{SUMMARY AND CONCLUSIONS}

To recapitulate briefly: (1) Apparatus was constructed which reduced the area of cross section of steel wire by wear over a length of about $60 \mathrm{~cm}$ with satisfactory uniformity.

(2) Observations were made to determine the effect of reduction of area by wear upon the magnetic properties and tensile strength of the wire.

(3) It was found that the effect on the magnetic properties, though much smaller in magnitude, was of the same nature as that of tensile stress, namely, an increase in permeability in the lower range of magnetizing force and a decrease in permeability for higher values of magnetizing force.

(4) The results indicated an increase in tensile strength per unit area with reduction in area, amounting to about 7 per cent for a reduction in area of 50 per cent.

From these results it was concluded that:

(1) The effect of wear on magnetic properties is due to a redistribution of internal stresses.

(2) As a result of the redistribution of internal stresses resulting from wear, a greater proportion of the remaining material becomes effective, thus accounting for the observed increase in tensile strength.

(3) With regard to the disturbing influence of wear on the results of magnetic exploration for the detection of flaws, since this effect is of the same nature and of much less magnitude, it can be treated in the same way as the effect of stress, namely, by making tests at properly chosen values of magnetizing force.

Washington, May 5, 1925. 